\title{
RELAÇ̃̃o ENTRE A QUALIDADE DA GESTÃo FISCAL E A TRANSPARÊNCIA DOS MUNICÍPIOS BRASILEIROS
}

RELATIONSHIP BETWEEN FISCAL MANAGEMENT QUALITY AND BRAZILIAN'S MUNICIPALITIES TRANSPARENCY

RELACIÓN ENTRE LA CALIDAD DE LA GESTIÓN FISCAL Y LA TRANSPARENCIA DE LOS MUNICIPIOS BRASILEÑOS

\section{RESUMO}

0 objetivo deste trabalho foi analisar a relação entre a qualidade da gestão fiscal e a transparência nos municípios brasileiros. Para tanto, foi realizado um estudo de caráter descritivo e quantitativo. Os dados foram secundários dos anos de 2015 e 2016.0 indicador de qualidade da gestão fiscal utilizado foi o Índice Firjan de Gestão Fiscal (IFGF); para transparência, foi utilizado 0 índice de transparência do Ranking Nacional de Transparência divulgado pelo Ministério Público Federal. As variáveis de controle foram Índice de Desenvolvimento Municipal, população, receita orçamentária bruta per capita, bem como dummies de tamanho e produtividade e arrecadação. A análise descritiva das variáveis de interesse da pesquisa identificou indicadores de gestão fiscal em níveis de dificuldade e transparência médios, heterogeneidade no tamanho, receita per capita e na transparência municipal. Por meio do modelo de regressão de mínimos quadrados generalizados (MQG), foi evidenciada uma relação positiva e significativa entre o indicador de qualidade de gestão pública municipal (IFGF) com todas as variáveis do modelo, validando 0 argumento teórico de que há influência dessas variáveis no indicador de qualidade de gestão pública municipal do Brasil. Portanto, aceita-se a hipótese geral da pesquisa ao se afirmar que a qualidade na gestão fiscal (IFGF) apresentou relação com a transparência dos municípios brasileiros e que a transparência municipal, Índice de Desenvolvimento Municipal, população, receita orçamentária bruta per capita, bem como dummies de tamanho e produtividade e arrecadação, estão relacionados com as variações do indicador de gestão fiscal.

PALAVRAS-CHAVE: Gestão fiscal, transparência, municípios brasileiros, accountability, gestão pública.

Geraldo Alemandro Leite Filho'

geraldo.alemandro@unimontes.br

ORCID: 0000-0003-2908-7907

Claudia Ferreira Cruz $^{2}$

claudiacruzba@gmail.com

ORCID: 0000-0001-5238-2029

João Paulo de Brito Nascimento²

jpbritonascimento@gmail.com

ORCID: 0000-0002-4986-4702

Tatiane Gomes Silva²

tatianegsilva@gmail.com

ORCID: 0000-0003-1484-5189

${ }^{1}$ Universidade Estadual de Montes Claros, Montes Claros, MG, Brasil

2 Universidade Federal do Rio de Janeiro, Rio de Janeiro, RJ, Brasil

Submetido 14.06.2018. Aprovado 23.11.2018

Avaliado pelo processo de double blind review. Editores convidados: Arnaldo Mauerberg Jr., José Roberto Afonso e Sol Garson.

DOI: http://dx.doi.org/10.12660/cgpc.v23n76.75408

Esta obra está submetida a uma licença Creative Commons 
Geraldo Alemandro Leite Filho - Claudia Ferreira Cruz - João Paulo de Brito Nascimento - Tatiane Gomes Silva

\section{ABSTRACT}

The aim of this work was to analyze the relationship between the quality of fiscal management and transparency in Brazilian municipalities. For that, a descriptive and quantitative study was carried out. Data were secondary for the years 2015 and 2016. The quality indicator for fiscal management used was FIRJAN's IFGF, while for transparency the transparency index of the National Transparency Ranking published by the Federal Public Prosecutor's Office was used. Control variables were municipal development index, population, gross budget revenue per capita as well as dummies of size and productivity and collection. The descriptive analysis of the variables of interest for the research identified indicators of fiscal management in levels of difficulty and average transparency, heterogeneity in size, per capita income and municipal transparency. The generalized least squares regression model (MQG) showed positive and significant relationship between the municipal public management quality indicator (IFGF) and all variables of the model, validating the theoretical argument that there is influence of these variables in the indicator quality of municipal public management in Brazil. Therefore, the general hypothesis of the research is corroborated when affirming that quality in fiscal management (IFGF) was related to the transparency of the Brazilian municipalities and that municipal transparency, municipal development index, population, gross budget revenue per capita as well as dummies of size and productivity and collection are related to the variations of the fiscal management indicator.

KEYWORDS: Fiscal management, transparency, Brazilian municipalities, accountability, public management.

\section{RESUMEN}

El objetivo de este trabajo fue verificar la relación existente entre la calidad de la gestión fiscal y la transparencia en los municipios brasileños. Para esto, se realizó un estudio de carácter descriptivo y cuantitativo. Los datos fueron secundarios y compréndió los años de 2015 y 2016. El indicador de calidad de la gestión fiscal utilizado fue el IFGF de FIRJAN, para la transparencia se utilizó el índice de transparencia del Ranking Nacional de Transparencia públicado por el Ministerio Público Federal. Las variables de control fueron índices de desarrollo municipal, población ingreso presupuestario bruta per cápita as í como dummies de tamaño y productividad y recaudación. El análisis descriptivo de las variables de interés de la investigación identificó indicadores de gestión fiscal en niveles de dificultad y transpparencia medianos, heterogeneidad en el tamaño, ingreso per cápita y en la transparencia municipal. Por medio del modelo de regresión de mínimos cuadrados generalizados (MQG) se evidenció una relación positiva y significativa entre el indicador de calidad de gestión pública municipal (IFGF) con todas las variables del modelo, validando el argumento teórico que hay influencia de esas variables en el indicador de calidad de gestión pública municipal de Brasil. Por lo tanto, se acepta la hipótesis general de la investigación al afirmar que la calidad en la gestión fiscal (IFGF) presentó relación con la transparencia de los municipios brasileños y que la transparencia municipal, índice de desarrollo municipal, población, ingreso presupuestario bruto per cápita as í como los dummies de tamaño y productividad y recaudación están relacionados con las variaciones del indicador de gestión fiscal.

PALABRAS CLAVE: Gestión fiscal, transparencia, municipios brasileños, accountability, gestión pública.

\section{INTRODUÇÃO}

Pequenos ou maiores, os municípios possuem importantes responsabilidades a cumprir, nos seus papéis de promoção da qualidade de vida de seus cidadãos (Klering, Kruel, \& Stranz, 2012). No entanto, a história recente evidencia que os municípios brasileiros enfrentaram e enfrentam diversos problemas, como recessão econômica, carência de recursos para investimentos, alto índice de corrupção e a ausência de ferramentas eficazes de planejamento e controle dos gastos públicos municipais. Considera-se que esses fatores impactam negativamente a gestão e o desempenho econômico e social desses entes federativos.

Segundo Zuccolotto e Teixeira (2014), por meio de uma administração transparente, o nível de confiança da população e do mercado no governo pode aumentar e promover maior estabilidade da economia e auxiliar a melhoria da gestão pública. Ainda segundo os autores, as democracias atuais estão associadas a uma gestão governamental trans- 
parente, então não há democracia efetiva sem transparência e acesso à informação por parte da sociedade. Portanto, uma gestão de qualidade deve estar alinhada com a transparência.

No Brasil, a Lei Complementar n. 101, de 04 de maio de 2000, conhecida como Lei de Responsabilidade Fiscal (LRF), representa um marco em relação à qualidade da gestão fiscal e tem, entre seus pilares, a transparência, uma vez que não é possível a participação social se não houver acesso à informação útil. No entanto, apesar de passados 18 anos de vigência, ainda se verifica que não há atendimento pleno às exigências de divulgação de informações públicas, bem como há casos de descumprimento de regras fiscais por municípios, como verificado por Piccoli e Arpini (2018) e Rodrigues e Salgueiro (2015).

Assim, sendo o município o ente governamental mais próximo do cidadão, conforme disposto por Ckagnazaroff (1989), e sendo a transparência fiscal algo fundamental para uma política econômica sólida, conforme Kopits e Craig (1998), o presente estudo tem como objetivo verificar a relação existente entre a qualidade da gestão fiscal e a transparência nos municípios brasileiros, de modo a identificar se resultados positivos na gestão fiscal são acompanhados de atuação governamental mais transparente.

Dessa forma, este estudo poderá contribuir empiricamente nas discussões que envolvem a relação entre qualidade na gestão fiscal e transparência dos municípios brasileiros e colaborar com os estudos alinhados à contabilidade aplicada ao setor público.
Para atingir o objetivo proposto, o presente estudo está estruturado com as seguintes seções, além da presente introdução: fundamentação teórica, que apresenta uma discussão sobre gestão fiscal municipal, transparência e estudos relacionados; posteriormente, são apresentados os procedimentos e métodos utilizados na pesquisa, bem como os resultados encontrados. Por fim, são apresentadas as considerações finais.

\section{FUNDAMENTAÇÃO TEÓRICA}

\section{Transparência na gestão pública}

A informação é essencial para o desenvolvimento de competências democráticas básicas, como a formulação de preferências e opiniões, a conjuntura de hipóteses e a participação na tomada de decisões. Sem essas competências, são negados a voz ao cidadão e o exercício de seus direitos (Klein \& Klein, 2017).

Considera-se que uma gestão transparente disponibiliza dados sobre as ações adotadas e resultados observados, de modo a demonstrar a sua atuação e permitir a participação da sociedade em geral.

A transparência nas operações do governo é amplamente considerada uma pré-condição importante para a sustentabilidade fiscal macroeconômica, boa governança e retidão fiscal geral (Kopits \& Craig, 1998).

Além disso, verifica-se a transparência como um importante instrumento para a avaliação da gestão fiscal, visto que constitui um dos pilares da responsabilidade fiscal. Nesse sentido, Cruz, Silva e Santos (2009) desta- 
caram a transparência da gestão fiscal como forma de promover uma melhor interação entre governo e os cidadãos, além de facilitar o exercício da cidadania e da accountability, que tem como um de seus aspectos a prestação de contas pelos gestores.

As iniciativas de transparência na administração pública constituem uma política de gestão responsável que favorece o exercício da cidadania pela população (Platt, Cruz, Ensslin, \& Ensslin, 2007). No entanto, em pesquisas relativas à transparência, é resultado comum a conclusão de divulgação incipiente de informações fiscais à sociedade pelos municípios, a exemplo de Bernardo, Reis e Sediyama (2017), Avelino, Cunha, Lima e Colauto (2014), Ribeiro e Zuccolotto (2009, 2012), Cruz, Ferreira, Silva e Macedo (2012), Sacramento e Pinho (2007) e Lock (2003). Logo, apesar da evolução na divulgação de informações, o nível de disponibilização dos dados em portais, mesmo em diferentes estratégias de investigação, ainda apresenta limitações.

Considerando a importância da gestão transparente, tem-se como referencial o Ranking Nacional de Transparência divulgado pelo Ministério Público Federal (MPF), elaborado a partir da verificação do atendimento a 16 perguntas relativas à divulgação de informações pelos municípios, considerando os seguintes aspectos: informações gerais; receita; despesa; licitações e contratos; relatórios (de gestão e obrigatórios definidos pela LRF) e possibilidade de exportação dos dados; transparência passiva; disponibilidade de serviço de informações ao cidadão; divulgação de estrutura e forma de contato; e boas práticas de transparência (MPF, 2017).
Entre os elementos de transparência, o planejamento e os resultados da gestão fiscal ganharam posição de destaque na divulgação de informações, especialmente a partir da LRF, conforme disposto a seguir.

\section{Gestão fiscal municipal}

A LRF é considerada o mais importante marco regulatório fiscal brasileiro (Kelles, 2010). A lei definiu metas relacionadas ao desempenho fiscal, relativas aos resultados entre receitas e despesas, além de limites e condições no que tange à renúncia de receita, geração de despesas com pessoal, da seguridade social e outras, dívidas consolidada e mobiliária, operações de crédito, inclusive por antecipação de receita, concessão de garantia e inscrição em restos a pagar. Além disso, os relatórios relativos à execução orçamentária e à gestão fiscal tiveram periodicidade definida e obrigatoriedade de divulgação, indicando a associação entre a transparência das informações e o desempenho fiscal.

De maneira mais ampla, entende-se a gestão fiscal como um dos aspectos de potencial interesse da sociedade, uma vez que representa o volume de arrecadação e o consequente emprego pelo governo dos recursos arrecadados da sociedade. Porém, para a análise de informações, é indispensável que os dados estejam disponíveis de modo acessível.

Diversas pesquisas abordaram a qualidade da gestão fiscal, considerando diferentes variáveis explicativas. A partir de Cruz et al. (2012), Ribeiro e Zuccoloto (2012) e Zuccoloto e Teixeira (2014), tem-se a dimensão da 


\section{RELAÇÃo ENTRE A QUALIDADE DA GESTÃo FISCAL E A TRANSPARÊNCIA DOS MUNICÍPIOS BRASILEIROS}

capacidade fiscal observada, considerando-se a receita orçamentária; a receita corrente; o Índice de Responsabilidade Fiscal, Social e Gestão dos municípios (IRFS); os gastos sociais; o resultado fiscal do governo e o endividamento. Aspectos relacionados ao desenvolvimento socioeconômico tiveram como variáveis explicativas o Produto Interno Bruto (PIB), Índice Firjan de Desenvolvimento Municipal - IFDM (emprego e renda), urbanização, educação e dinamismo municipal, somando aos anteriores os estudos de Wright e Paulo (2014) e Reis, Ferreira e Ferreira (2015).

Para contribuir com uma gestão pública eficiente e democrática, o Sistema Firjan desenvolveu o Índice Firjan de Gestão Fiscal (IFGF). O IFGF propõe-se a ser uma ferramenta de controle social como objetivo de estimular a cultura da responsabilidade administrativa, possibilitando maior aprimoramento da gestão fiscal dos municípios, bem como o aperfeiçoamento das decisões dos gestores públicos quanto à alocação dos recursos (Federação das Indústrias do Estado do Rio de Janeiro - Firjan, 2017b).

Nesse contexto, o IFGF agrega informações municipais sobre receita própria, gastos com pessoal, investimentos, liquidez e custo da dívida, sendo pontuado de 0 a 1, e considera como boa gestão fiscal o desempenho a partir de 0,6 pontos.

O IFGF será considerado na presente pesquisa como o indicador de qualidade na gestão fiscal, priorizando-se a abrangência dos seus componentes, que consideram, além de parâmetros da LRF, medida de geração de receita (receita própria), a suficiência dos recursos para honrar os compromissos (Ii- quidez) e o custo relacionado ao endividamento (custo da dívida).

Accountability theory como resposta à assimetria informacional

Não há como os cidadãos e entidades civis acompanharem, conhecerem, controlarem a utilização de recursos se não houver acesso a informação útil (Silva, 2009).

A assimetria informacional é tratada pela Teoria da Agência no âmbito do conflito entre principal e agente. Dada a diferença de informações disponíveis na relação entre governo (agente) e sociedade (principal), uma forma de diminuir tal lacuna é a prática de accountability, processo que envolve a obrigatoriedade de que o poder seja exercido de modo transparente, com as correspondentes prestações de contas e sujeito à aplicação de sanções.

O termo accountability concentraria a preocupação com verificações e fiscalização para vigilância e restrições institucionais sobre o exercício do poder. Schedler (1999, p. 13-14) descreveu três formas de prevenir e corrigir o abuso do poder político: sujeitar o poder à ameaça de sanções, estabelecer a obrigatoriedade da atuação transparente e forçá-lo a justificar os seus atos, ações representadas por enforcement, monitoring e justification. Ou seja, é necessário a atuação transparente por parte dos gestores, que estes sejam passíveis de sanções por atos inadequados e que sua atuação ocorra de modo a demonstrar e justificar as escoIhas realizadas.

Campos (1990) destacou que o grau de accountability de uma determinada demo- 
cracia é explicado pelas dimensões do macroambiente da administração pública: a textura política e institucional da sociedade; os valores e os costumes tradicionais partilhados na cultura; a história. A autora destacou que, quanto menos amadurecida a sociedade, menos provável que se preocupe com a accountability do serviço público. Por fim, verificou a impossibilidade, na época, de tradução do termo para o português. Posteriormente, Pinho e Sacramento (2009) reconheceram a existência de avanços no sentido de mudanças no cenário brasileiro, especialmente quanto à organização da sociedade, descentralização e transparência governamental e quanto à emergência de novos valores sociais em substituição aos tradicionais, porém ainda muito longe de construir uma verdadeira cultura de accountability.

Recentemente, o termo consolidou-se como teoria, a Accountability Theory (AT), que abrange o processo no qual os indivíduos têm a obrigação potencial de explicar as suas ações a outros indivíduos, os quais têm o direito de julgar as ações e administrar consequências positivas ou negativas em resposta a essas ações (Klein \& Klein, 2017).

Assim, o presente estudo está embasado pela Accountability Theory e investiga a relação entre a qualidade na gestão fiscal e a transparência nos municípios, considerando a divulgação de informações como ferramenta para viabilizar o controle social e fortalecimento de uma cultura de accountability no País.

\section{Estudos relacionados}

A pesquisa sobre transparência apresenta-se mais ampla em relação aos estudos relativos à qualidade da gestão fiscal de municípios. Oliveira e Santos (2017), ao estudarem a transparência passiva em pequenos municípios, apresentaram a expectativa teórica de que municípios com maior capacidade fiscal apresentassem também melhores índices de transparência passiva, pois estudos anteriores, a exemplo de Ribeiro e Zuccolotto (2012) e Cruz et al. (2012), indicaram que a transparência administrativa está associada à melhoria na gestão fiscal. Os autores concluíram que os pequenos municípios brasileiros com maior capacidade fiscal apresentam também melhores índices de transparência passiva.

Wright (2013), por sua vez, realizou estudo destinado a identificar os fatores determinantes da transparência fiscal ativa nos governos municipais brasileiros. Especificamente no tocante à divulgação, os resultados sugeriram a existência de uma maior dificuldade para a disponibilização da transparência fiscal ativa por parte dos municípios com população menor que 50 mil habitantes, bem como para os municípios situados nas regiões Norte e Nordeste do País, demonstrando a existência de diferença de desempenho considerando-se a região geográfica. Na mesma linha, Barros (2014) apontou que os maiores municípios têm transparência fiscal mais efetiva, enquanto aqueles com menos de 50 mil habitantes apresentam dificuldades em divulgar seus dados, como resultado da análise dos portais de transparência dos 399 municípios do estado do Paraná.

Em relação aos determinantes para a maior transparência, Cruz et al. (2012) e Ribeiro e Zuccolotto (2012) verificaram que municípios 
com maior arrecadação relativa (receita orçamentária per capita) tendem a divulgar mais informações em meio eletrônico de acesso público, devido à disponibilidade de recursos para investimento na gestão da informação. Cruz et al. (2012) identificaram, ainda, relações positivas e significativas entre nível de transparência fiscal eletrônica e as variáveis população, taxa de alfabetização, Índice de Desenvolvimento Humano Municipal (IDH-M), IFDM e estágio do site.

Cruz, Macedo e Sauerbronn (2013), ao realizarem estudo com grandes municípios, considerando o período de 2002 a 2009, em pesquisa voltada à análise das características que influenciam a responsabilidade fiscal de grandes municípios brasileiros, verificaram que os municípios brasileiros pesquisados apresentam um nível incipiente de responsabilidade fiscal.

Recentemente, Cruz e Afonso (2018) analisaram a relação entre indicadores de metas e limites fiscais com variáveis representativas dos pilares de planejamento, transparência e controle, considerando 282 municípios brasileiros com população superior a 100 mil habitantes, no período de 2010 a 2013, tendo concluído que os indicadores fiscais não apresentaram relações significativas entre si e revelaram reduzida associação com outras variáveis representativas da gestão fiscal.

A relação entre desenvolvimento e transparência também é objeto de estudo, habitualmente englobando questões como Índice de Desenvolvimento Municipal (IDM), população, receita arrecadada, PIB, região do município e localização (capital, região metropolitana ou interior).
Rodrigues e Salgueiro (2015) observaram o IDM como a característica que mais pode influenciar na melhor divulgação do município, entretanto o coeficiente de correlação de Pearson apontou que o grau de correlação não é forte, ao estudarem os municípios do Ceará.

Cruz et al. (2012), ao verificarem as informações divulgadas nos portais eletrônicos de 96 dos 100 municípios mais populosos do Brasil, concluíram que, no geral, existe associação entre as condições socioeconômicas dos municípios e os níveis de transparência na divulgação de informações acerca da gestão pública.

Comin, Ramos, Zucchi, Favretto e Fachi (2016) buscaram identificar a relação existente entre o índice de atendimento à Lei de Acesso à Informação (LAI) dos municípios catarinenses e os seus indicadores socioeconômicos (população total, receita arrecadada, PIB e IDH-M). Como resultado, verificaram que nenhum dos municípios atende a todas as exigências da LAI e, também, a existência de relação positiva do índice de atendimento apenas com a variável IDH-M.

No tocante à relação entre gestão fiscal e desenvolvimento ou qualidade de vida, há diferentes estudos, sob a temática da LRF, gestão fiscal, gasto público e políticas públicas, que buscaram verificar a existência de relação entre realização de investimentos ou gestão fiscal e a qualidade de vida da população.

Leite, Santiago, Otoni, Veloso e Ferreira (2015), ao verificarem relação entre indicadores de premissas da LRF (IRFS), de 
responsabilidade social municipal (IMRS) e de desenvolvimento (IFDM) dos municípios de Minas Gerais, considerando o período de 2005 a 2010, constataram que a variável IRFS Fiscal apresentou maior coeficiente significativo e positivo nessa relação, sugerindo que os aspectos fiscais foram mais expressivos na explicação da variação do indicador de desenvolvimento dos municípios. Concluiu-se, também, ter havido efeitos positivos e significativos entre 0 cumprimento das prerrogativas da LRF e a responsabilidade social. Foram verificadas diferenças consideráveis na explicação da relação entre o IRFS, IFDM e IMRS, quando analisadas as mesorregiões do estado de Minas Gerais.

No tocante à associação entre responsabilidade fiscal e transparência, especificamente considerando o IFGF, foram encontradas poucas pesquisas acadêmicas divulgadas. Destacam-se os estudos de Avelino (2013) e Bernardo et al. (2017), que tiveram o IFGF como uma das variáveis independentes, entre outras que foram avaliadas no intuito de verificar determinantes de transparência.

Avelino (2013) verificou que, conforme aumentava o IFGF do município, também aumentava a probabilidade de o município apresentar um grau de disclosure considerado bom em vez de ruim, ou seja, municípios com altos valores de IFGF tenderiam a divulgar mais informações.

Os resultados de Ribeiro e Zuccolotto (2012) e Avelino (2013), entretanto, são diferentes do encontrado por Bernardo et al. (2017), que identificaram comportamento negativo do IFGF em relação à transparência, porém de resultado estatisticamente não significante.

No entanto, destaca-se que são reduzidas as pesquisas que avaliam o desempenho dos municípios relacionando o IFGF e a transparência. Dessa forma, o presente trabalho propõe-se a suprir essa lacuna, com a realização de análise dos municípios brasileiros com informações disponíveis, e concentra-se na avaliação da relação entre IFGF e a transparência dos municípios brasileiros.

\section{PROCEDIMENTOS E MÉTODOS DA PES- QUISA}

\section{Caracterização e procedimentos}

Este estudo caracteriza-se como um estudo de avaliação quantitativa, quanto à abordagem, e descritivo, que tem por objetivo a coleta de dados de uma população ou amostra para verificação de hipóteses previamente estabelecidas. Os dados utilizados nesta pesquisa são de caráter secundário e têm as suas características apresentadas no Quadro 1. 


\section{RELAÇÃO ENTRE A QUALIDADE DA GESTÃo FISCAL E A TRANSPARÊNCIA DOS MUNICÍPIOS BRASILEIROS}

Quadro 1. Características dos dados utilizados na pesquisa

\begin{tabular}{|c|l|l|c|}
\hline Dimensão & \multicolumn{1}{|c|}{ Variável } & \multicolumn{1}{|c|}{ Base } & Período \\
\hline Gestão Fiscal & $\begin{array}{l}\text { Índice Firjan de Gestão Fiscal } \\
\text { (IFGF) }\end{array}$ & $\begin{array}{l}\text { Federação das Indústrias do Estado } \\
\text { do Rio de Janeiro (Firjan) }\end{array}$ & 2015 e \\
\hline Transparência & Índice de Transparência & Ministério Público Federal (MPF) & 2016 \\
\hline Socioeconômica & $\begin{array}{l}\text { Índice Firjan de Desenvolvimento } \\
\text { Municipal - IFDM }\end{array}$ & Firjan & $\begin{array}{l}\text { Instituto Brasileiro de Geografia e } \\
\text { Estatísticas (IBGE) }\end{array}$ \\
\hline Tamanho & População Estimada & Tesouro Nacional - Siconfi & \\
\hline Econômica & $\begin{array}{l}\text { Receita Orçamentária Bruta Reali- } \\
\text { zada }\end{array}$ & &
\end{tabular}

O período analisado foi estabelecido em dois anos, 2015 e 2016, devido à disponibilidade de dados do Ranking Nacional de Transparência, divulgado pelo MPF, que realizou avaliação quanto à observância dos dispositivos legais de transparência nesses dois anos.

A população-alvo da pesquisa englobou 5.568 municípios brasileiros, dessa forma foram desconsiderados Brasília e Fernando de Noronha, que são alocados em distritos e apresentam características políticoadministrativas distintas (Cruz et al., 2013). Destaca-se, ainda, que esses dois distritos não compõem as bases da Firjan e do MPF.

Como critério na organização dos dados, foi definido que os municípios deveriam ter dados válidos nos anos pesquisados $e$ em todas as variáveis. Dessa forma, foram excluídos 1.165 municípios que não apresentaram os dados nos dois períodos simultaneamente, o que gerou uma amostra de 4.403 municípios para cada ano. Essa amostra correspondeu a $79,05 \%$ do total dos municípios brasileiros.

\section{Descrição das variáveis do modelo}

A dimensão gestão fiscal está representada pelo IFGF, que é destinado a avaliar a qualidade da gestão fiscal dos municípios brasileiros. O IFGF é composto por cinco indicadores: receita própria, gastos com pessoal, investimentos, liquidez e custo da dívida (Firjan, 2017a).

A partir desse contexto e com o objetivo de ampliar ao máximo as observações na análise da existência de relação entre a qualidade da gestão fiscal e transparência, foi utilizado como parâmetro da dimensão transparência municipal o Ranking Nacional de Transparência divulgado pelo MPF (2017), excluídas as informações sobre os estados.

Com o objetivo de tornar o modelo mais explicativo, direcionados pelos estudos de Bernardo et al. (2017), Cruz (2010), Cruz et al. (2012), Leite et al. (2015), Oliveira e Santos (2017) e Ribeiro e Zuccolotto (2009, 2012), foram selecionadas as seguintes variáveis explicativas e/ou controle: 
a) Desenvolvimento municipal (IFDM): índice desenvolvido pela Firjan que avalia o desenvolvimento socioeconômico de todos os municípios brasileiros, sendo uma função de três subíndices: emprego e renda, educação e saúde.

b) Receita orçamentária bruta realizada per capita (RecPCap): a receita orçamentária bruta realizada são os recursos econômicos geridos pelos municípios. Para representação dessa dimensão econômica, foi utilizada a receita orçamentária per capita de cada município.

c) População (Pop): população estimada pelo IBGE para os anos de 2015 e 2016. Essa variável está relacionada ao tamanho dos municípios por habitantes.

d) Capacidade produtiva e arrecadação $\left(D_{-}\right.$ ProdArrec): variável dummy, sendo 1 para grupo de municípios com capacidade produtiva e arrecadação acima da média e 0 para municípios abaixo da média. Utilizou-se como base para cálculo da média a receita bruta orçamentária realizada.

e) Tamanho (D_Tam): variável dummy para dois grupos de municípios, sendo 1 para municípios com população maior que 50 mil habitantes e 0 para municípios com população até 50 mil habitantes.

Ressalta-se que, neste estudo, optou-se pela utilização dos dados sem tratamentos de ajustes.

\section{Modelo e hipótese do estudo}

Considerando que o objetivo do trabalho é verificar a relação existente entre a qualidade na gestão fiscal e a transparência, foi estruturado o modelo geral de regressão, que é representado pela seguinte equação:

\section{IFGF $=\beta \_0+\beta \_1$ Transp $+\beta \_2$ IFDM $+\beta \_3$ RecPCap $+\beta \_4$ \\ $T a m+\beta \_6$ Pop $+\mathcal{E}$ D_ProdArrec+ $+\beta \_5$ \\ (1)}

Ponderando sobre a limitação temporal dos dados deste estudo, que são de dois anos, 2015 e 2016, portanto uma série curta, optou-se pelo modelo para dados empilhados (pooled data). Essa escolha está orientada por posicionamento semelhante de Washington, Karlaftis e Mannering (2011) e questionamentos de Beck e Katz (2011), quando discutiram sobre utilizar modelos baseados em painel ou corte transversal quando a série temporal é curta. Esses autores apontam que, para séries curtas, a opção por modelos de regressão por dados empilhados pode ser adotada.

Dessa forma, tem-se como hipótese geral deste estudo:

H1: Existe relação entre a gestão fiscal municipal e a transparência.

Considerando os estudos anteriores de Avelino (2013), Avelino et al. (2014), Bernardo et al. (2017), Cruz et al. (2012, 2013), Leite et al. (2015), Ribeiro e Zuccolotto (2009, 2012), espera-se que as variáveis explicativas apresentem relação positiva com a qualidade na gestão fiscal.

\section{RESULTADOS E DISCUSSÃO}

Análise descritiva e de correlação das variáveis

A Tabela 1 apresenta a estatística descritiva dos dados empilhados dos municípios brasileiros que compõem a amostra da pesquisa. 
RELAÇÃo ENTRE A QUALIDADE DA GESTÃo FISCAL E A TRANSPARÊNCIA DOS MUNICÍPIOS BRASILEIROS

Tabela 1. Testes dos pressupostos da regressão

\begin{tabular}{|l|c|c|c|}
\hline \multicolumn{1}{|c|}{ Pressupostos } & Teste & Estatística & p-valor \\
\hline Normalidade & Qui-quadrado & 24,6656 & $<0,0000$ \\
\hline Homocedasticidade & White (LM) & $>253,682$ & $<0,0000$ \\
\hline Multicolinearidade & \multicolumn{3}{|c|}{} \\
& Fator de Inflação de Variância (FIV) & \\
& Transp & 1,129 & \\
& IFDM & 1,268 & \\
& RecPCap & 1,157 & \\
& Pop & 1,107 & \\
& D_ProdArrec & 3,360 & \\
& D_Tam & 3,362 & \\
\hline
\end{tabular}

Com relação às médias, verificou-se que a variável dependente da pesquisa, o indicador de gestão fiscal municipal, apresentou média de 0,4554, mostrando um panorama médio de gestão em dificuldade $(0,4<0,6)$ para os municípios brasileiros no período de 2015 a 2016. Tais resultados são condizentes com os estudos anteriores que estudaram variáveis de gestão fiscal, a saber: Cruz et al. (2012); Ribeiro e Zuccoloto (2012); Zuccoloto e Teixeira (2014); Wright e Paulo (2014) e Reis et al. (2015).

O resultado médio da variável independente, o indicador de transparência, apresentou média de 4,8 em um intervalo de zero a 10 , para os municípios brasileiros no período de 2015 a 2016, que pode ser considerado um nível baixo de transparência. Tais resultados são compatíveis com os estudos de Bernardo et al. (2017), Avelino et al. (2014), Ribeiro e Zuccolotto (2009, 2012), Cruz et al. (2012), Sacramento e Pinho (2007) e Lock (2003), nos quais os autores observaram uma divulgação incipiente de informações fiscais à sociedade pelos municípios brasileiros.

Com base na estatística descritiva, observaram-se as seguintes dispersões em termos de coeficiente de variação, a saber: tamanho em termos de população (576\%) e receita per capita (48\%), índice de gestão fiscal apresentou uma variabilidade de $29 \%$ e o indicador de transparência, 57\%. O indicador de desenvolvimento municipal, por sua vez, apresentou uma variabilidade de $17 \%$, considerada a menor de todas as variáveis, sugerindo uma homogeneidade em termos de indicador de desenvolvimento municipal.

De uma maneira geral, o quadro descritivo das variáveis de interesse da pesquisa acerca dos municípios brasileiros evidenciou significativa heterogeneidade em termos de tamanho (população), receita per capita e na transparência municipal. A qualidade da gestão fiscal apresentou menor dispersão, sugerindo que, embora outros contextos sejam diferentes, a qualidade da gestão segue uma certa homogeneidade na amostra, as- 
sim como o indicador de desenvolvimento municipal.

Por meio da análise de correlação, que é apresentada na Tabela 2, buscou-se evidenciar a associação entre as variáveis da pesquisa.

Tabela 2. Estatísticas descritivas dos municípios

\begin{tabular}{lcccc}
\hline \multicolumn{1}{c}{ Variável } & Média & Mínimo & Máximo & Desvio-Padrão \\
IFGF & 0,45545 & 0,066917 & 0,95697 & 0,13352 \\
Transp & 4,8003 & 0,00000 & 10,000 & 2,7283 \\
IFDM & 0,66538 & 0,00000 & 0,90394 & 0,11216 \\
RecPCap & 3397,4 & 250,84 & 23401 & 1618,8 \\
Pop & 37365,0 & 818,00 & 11.967 .825 & $215.260,00$ \\
D_ProdArrec & - & - & - & - \\
D_Tam & - & - & - & - \\
\hline
\end{tabular}

Nota: 8806 observações - dados empilhados

Observou-se que, para o período de análise (de 2015 a 2016), a qualidade da gestão pública relacionou-se de maneira fraca a moderada $(0,2<r<0,69)$, positiva e significativa a $5 \%$ com todas as variáveis de interesse da pesquisa, dando evidências de haver associação entre todos esses indicadores. Destaca-se a associação dessa variável com o indicador de desenvolvimento, receita per capita, transparência e variáveis dummies produtividade e arrecadação dos municípios e de tamanho. De uma maneira geral, tais resultados dão evidências de que a gestão fiscal municipal brasileira está associada com o conjunto de variáveis propostas neste trabalho, validando os trabaIhos de Zuccolotto e Teixeira (2014), Cruz et al. (2012), Oliveira e Santos (2017), Ribeiro e Zuccolotto (2012), Avelino (2013) e Cruz et al. (2009), nos quais os autores indicaram que a transparência está associada à melhoria na gestão fiscal, bem como os trabalhos de Leite et al. (2015), nos quais os autores identificaram que os níveis de desenvolvimento influenciaram o indicador de gestão fiscal.
Entre as outras variáveis do modelo, foram observadas correlações fracas $(r<0,39)$, excluindo-se apenas a relação forte $(0,7<r$ $<1$ ), positiva e significativa a $5 \%$ entre as variáveis dummy de tamanho e dummy de capacidade produtiva e arrecadação. Portanto, esse resultado indica uma relação de quanto maior o município em termos de população maior arrecadação e maior desenvolvimento, validando as proposições de Cruz et al. (2012, 2013), Ribeiro e Zuccolotto (2012) e Cruz e Afonso (2018), nos quais os autores argumentam sobre a influência do tamanho em população com variáveis de desenvolvimento, arrecadação e produtividade econômica.

Após as etapas de análise descritiva dos dados e análise da matriz de correlação das variáveis de interesse da pesquisa e observar que a qualidade da gestão fiscal está relacionada positiva e significativamente com as demais variáveis, foi processada regressão para verificar o efeito conjunto de tais variáveis na qualidade da gestão dos municípios 
com dados conjuntos dos anos de 2015 e 2016, conforme apresentado a seguir.

Análise do modelo de regressão

Para os modelos de regressão, os dados foram organizados no formato dados empilha- dos. Foram processadas regressões de estimativas por mínimos quadrados ordinários para que, em seguida, fossem realizados os testes dos pressupostos estatísticos relativos aos erros da regressão, cujos resultados são apresentados na Tabela 3.

Tabela 3. Matriz de correlação das variáveis da pesquisa

\begin{tabular}{lrrrrrrr}
\hline Variáveis & IFGF & Transp & IFDM & RecPCap & Pop & D_ProdArrec & D_Tam \\
\hline FGF & 1 & & & & & & \\
Transp & 0,2485 & 1 & & & & & \\
IFDM & 0,3260 & 0,3056 & 1 & & & & \\
RecPCap & 0,2835 & 0,1812 & 0,2729 & 1 & & & \\
Pop & 0,0930 & 0,0885 & 0,0968 & $-0,0441$ & 1 & & \\
D_ProdArrec & 0,1976 & 0,1559 & 0,2922 & $-0,0363$ & 0,2822 & 1 & \\
D_Tam & 0,1231 & 0,1306 & 0,2148 & $-0,1400$ & 0,3017 & 0,8280 & 1 \\
\hline
\end{tabular}

Nota: a) 8806 observações - dados empilhados b) Significativa a 5\%.

Observa-se que os resultados dos testes de especificação não validaram os modelos em termos de normalidade (teste Qui-quadrado, p-valor $<0,0000)$ e homocedasticidade (teste White (LM), p-valor $<0,0000$ ). No entanto, quanto à normalidade, assume-se o teorema do limite central para inferir que os resíduos tendem a uma normalidade assintótica, uma vez que a amostra pode ser considerada grande $(\mathrm{n}=8806$ observações), portanto não rejeitando a hipótese de normalidade do erro por aproximação (Gujarati \& Porter, 2011, p. 328; Wooldridge, 2006, p. 163). Em relação à homocedasticidade, a correção sugerida para que se possa ajustar os erros dos modelos de regressão é a aplicação de erros-padrão robustos (Gujarati \& Porter, 2011, p. 395).

A seguir, na Tabela 4, são apresentados os resultados das estimações das regressões, utilizando-se do pooled por MQG: 
Tabela 4. Resultados do modelo de regressão

\begin{tabular}{|c|c|c|c|c|}
\hline \multicolumn{5}{|c|}{ Pooled por Mínimos Quadrados Generalizados (MQG) } \\
\hline \multirow{2}{*}{\multicolumn{5}{|c|}{$\begin{array}{l}8806 \text { observações, incluídas } 4403 \text { unidades de corte transversal } \\
\text { Comprimento da série temporal = } 2\end{array}$}} \\
\hline & & & & \\
\hline \multicolumn{5}{|c|}{ Variável dependente: IFGF } \\
\hline Variável & Coef. & Erro-Padrão & Estatística t & p-valor \\
\hline Const & 0,209306 & 0,0114294 & 18,31 & $<0,0001^{* * *}$ \\
\hline Transp & 0,006401 & 0,000594389 & 10,77 & $<0,0001^{* * *}$ \\
\hline IFDM & 0,225212 & 0,0189816 & 11,86 & $<0,0001^{* * *}$ \\
\hline RecPCap & 0,000017 & 0,0000012 & 13,44 & $<0,0001^{* * *}$ \\
\hline Pop & 0,000000026 & 0,000000013 & 2,062 & $0,0393^{* *}$ \\
\hline D_ProdArr & 0,061180 & 0,00936361 & 6,534 & $<0,0001^{* * *}$ \\
\hline D_Tam & 0,020211 & 0,00994214 & 2,033 & $0,0421^{* *}$ \\
\hline \multicolumn{5}{|c|}{ Informações do modelo } \\
\hline$R^{2}: 0,182588$ & & \multicolumn{3}{|c|}{$\mathrm{R}^{2}$-ajustado: 0,182031 } \\
\hline Estatística F $(6,4$ & 384 & \multicolumn{3}{|c|}{ p-valor: $<0,000000$} \\
\hline
\end{tabular}

Nota: ${ }^{* * *}=$ sig. $<0,01 ;{ }^{* *}=$ sig. $<0,05$.

Observou-se que o modelo de regressão como um todo foi significativo $\mathrm{P}$-valor $(\mathrm{F})>$ 0,0001 e apresentou um coeficiente de determinação ( $R$ quadrado ajustado) no qual as variáveis explicaram conjuntamente $18,20 \%$ da variação do indicador de qualidade da gestão pública dos municípios.

A variável independente do modelo, a transparência, apresentou coeficiente positivo e significativo a $1 \%$, sugerindo que ela causou efeitos positivos na variação do indicador de gestão fiscal dos municípios brasileiros. Tais resultados confirmam o modelo teórico de que haveria esse efeito e validam os trabalhos de Bernardo et al. (2017), Avelino et al. (2014), Ribeiro e Zuccolotto (2009, 2012), Cruz et al. (2012), Sacramento e Pinho (2007), Lock (2003), Ribeiro e Zuccoloto (2012), Zuccoloto e Teixeira (2014), Wright e Paulo (2014) e Reis et al. (2015), que afirmam que níveis de transparência in- fluenciam a gestão fiscal dos municípios. $\mathrm{O}$ trabalho de Cruz et al. (2009) ainda advoga a transparência como um importante instrumento para a avaliação da gestão fiscal

A transparência da gestão fiscal municipal é uma questão não só de direito mas de exercício da cidadania, pois, segundo Zuccolotto e Teixeira (2014), não há democracia efetiva sem transparência e acesso à informação por parte da sociedade. Por outro lado, foi demonstrado, na análise descritiva dos dados, o baixo grau de transparência municipal, evidenciado pelos estudos de Piccoli e Arpini (2018) e Rodrigues e Salgueiro (2015) de que, em uma parte significativa dos municípios, não há atendimento pleno às exigências de divulgação de informações públicas, bem como há casos de descumprimento de regras fiscais por municípios, o que compromete ainda mais tal indicador. Entende-se, assim como em Kopits e Craig (1998), que 
a transparência deve ser considerada uma pré-condição importante para a sustentabilidade fiscal macroeconômica municipal, boa governança e retidão fiscal geral, contribuindo significativamente para o desempenho na gestão fiscal pública.

A variável de controle que capturou os efeitos do desenvolvimento dos municípios apresentou maior coeficiente positivo e significativo a $1 \%$ na relação da transparência com a qualidade da gestão pública municipal, evidenciando que o desenvolvimento municipal influencia a qualidade da gestão fiscal, corroborando o trabalho de Leite et al. (2015), no qual os autores ressaltam os efeitos dos indicadores de desenvolvimento nos indicadores de gestão fiscal dos municípios. Maiores níveis de desenvolvimento estariam relacionados a melhores indicadores de gestão fiscal.

A variável de controle que capturou os efeitos da receita bruta per capita apresentou coeficiente positivo e significativo a $1 \%$ na relação com a qualidade da gestão pública municipal, evidenciando que o efeito conjunto do tamanho e da produtividade e arrecadação influenciam a qualidade da gestão fiscal municipal. Tais resultados são compatíveis com as observações de Cruz et al. (2012, 2013), Ribeiro e Zuccolotto (2012), e Cruz e Afonso (2018), nas quais os autores advogam que municípios com maior arrecadação relativa (receita orçamentária per capita) tendem a divulgar mais informações em meio eletrônico de acesso público, devido à disponibilidade de recursos para investimento na gestão da informação, e esse aspecto influencia a gestão fiscal.

A variável de controle que capturou os efei- tos da capacidade produtiva e arrecadação dos municípios apresentou o segundo maior coeficiente positivo e significativo a $1 \%$ na relação da transparência com a qualidade da gestão pública municipal, evidenciando que o desenvolvimento, arrecadação e produtividade e infraestrutura influentes na capacidade produtiva e de arrecadação municipal influenciam a qualidade da gestão fiscal. Tais resultados corroboram Rodrigues e Salgueiro (2015), Cruz et al. (2012) e Comin et al. (2016), que destacam a associação existente entre as condições socioeconômicas dos municípios e os níveis de transparência na divulgação de informações acerca da gestão pública.

As demais variáveis de controle (população, dummy de tamanho) apresentaram coeficiente positivo e significativo a $5 \%$ na relação da transparência com a qualidade da gestão pública municipal, evidenciando que o tamanho tem efeitos positivos na relação transparência e gestão fiscal municipal. Nesse sentido, Wright (2013) e Barros (2014) sugeriram a existência de uma maior dificuldade para a disponibilização da transparência fiscal ativa por parte dos municípios com menos de 50 mil habitantes, demonstrando a existência de diferença de desempenho considerando-se o tamanho da população. Observaram, ainda, que municípios maiores apresentam melhor desempenho na gestão fiscal e têm transparência fiscal mais efetiva, enquanto aqueles com menos de 50 mil habitantes apresentam dificuldades em divulgar seus dados.

Os resultados encontrados na regressão corroboraram o que foi evidenciado na análise de correlação, uma vez que a associação entre $\mathrm{o}$ indicador de qualidade de gestão 
fiscal municipal e a transparência e as variáveis de controle (desenvolvimento municipal, tamanho, receita per capita e nível de arrecadação) foi positiva e estatisticamente significante. Isso reforça o argumento de que há influência de tais variáveis na relação da transparência e qualidade de gestão fiscal municipal do Brasil.

Nesse sentido, Zuccolotto e Teixeira (2014), Cruz et al. (2012), Oliveira e Santos (2017), Ribeiro e Zuccolotto (2012) e Avelino (2013) indicaram que a transparência administrativa está associada à melhoria na gestão fiscal, e os trabalhos sugeriram que municípios com maior índice de qualidade de gestão fiscal apresentam também melhores índices de transparência. Assim, conforme aumentava o índice de gestão fiscal do município, também aumentava a probabilidade de o município apresentar um grau de disclosure, ou seja, divulgava mais informações, corroborando a teoria da accountability (Klein \& Klein, 2017; Pinho \& Sacramento, 2009).

\section{CONCLUSÃO}

O objetivo do presente trabalho foi verificar a relação existente entre a qualidade da gestão fiscal e a transparência nos municípios brasileiros, de modo a identificar se resultados positivos na gestão fiscal são acompanhados de atuação governamental mais transparente.

De uma maneira geral, o quadro descritivo das variáveis de interesse da pesquisa acerca dos municípios brasileiros evidenciou a existência de heterogeneidade em termos de tamanho (população), receita per capita e na transparência municipal.
A qualidade da gestão fiscal apresentou menor dispersão, sugerindo que, embora outros contextos sejam diferentes, a qualidade da gestão segue uma certa homogeneidade na amostra, assim como o indicador de desenvolvimento municipal. Os indicadores médios de transparência foram considerados baixos, e o indicador médio de qualidade da gestão fiscal municipal foi classificado como gestão em dificuldade.

Concluiu-se que a qualidade da gestão fiscal dos municípios apresentou relação de maneira fraca a moderada, positiva e significativa com todas as variáveis de interesse da pesquisa, dando evidências de haver associação entre todas as variáveis. A variável independente do modelo, a transparência, apresentou coeficiente positivo e significativo a $1 \%$, sugerindo que ela causou efeitos positivos na variação do indicador de gestão fiscal dos municípios brasileiros. Concluiu-se, assim, que níveis de transparência estão associados à qualidade da gestão fiscal dos municípios.

Os resultados encontrados na regressão corroboraram os resultados da análise de correlação e sugerem a conclusão de que o indicador de qualidade de gestão fiscal municipal sofre efeitos da transparência e com as variáveis de controle (desenvolvimento municipal, tamanho, população, receita per capita e nível de produtividade e arrecadação), reforçando o argumento de que há influência dessas variáveis na relação transparência e qualidade de gestão fiscal municipal no Brasil. Eles também permitem concluir que resultados positivos na gestão fiscal são acompanhados de atuação governamental municipal mais transparente. 


\section{RELAÇÃO ENTRE A QUALIDADE DA GESTÃO FISCAL E A TRANSPARÊNCIA DOS MUNICÍPIOS BRASILEIROS}

\section{REFERÊNCIAS}

Avelino, B. C. (2013). Características explicativas do nível de disclosure voluntário de municípios do estado de Minas Gerais: Uma abordagem sob a ótica da teoria da divulgação (Dissertação de mestrado, Universidade Federal de Minas Gerais, Belo Horizonte, MG).

Avelino, B. C., Cunha, J. V. A., Lima, G. A. S. F., \& Colauto, R. D. (2014). Características explicativas do nível de disclosure voluntário de municípios do estado de Minas Gerais. Revista de Administração, Contabilidade e Economia, 13(2), 571-606.

Barros, L. M. (2014). A transparência fiscal eletrônica nos municípios do estado do Paraná: Avaliação do índice de transparência e as possíveis relações nesse processo (Dissertação de mestrado, Universidade Federal do Paraná, Curitiba, PR).

Beck, N., \& Katz, J. N. (2011). Modeling dynamics in time-series-cross-section political economy data. Annual Review of Political Science, 14, 331-352. doi:10.1146/annurev-polisci-071510-103222

Bernardo, J. S., Reis, A. O., \& Sediyama, G. A. S. (2017). Características explicativas do nível de transparência na administração pública municipal. Revista Ciências Administrativas, 23(2), 277-292. doi:10.5020/23180722.23.2.277-292

Campos, A. M. (1990). Accountability: Quando poderemos traduzi-la para o português? RAP-Revista de Administração Pública, 24(2), 30-50.
Ckagnazaroff, I. B. (1989). A Nova Constituição: Uma nova administração municipal. RAP-Revista de Administração Pública, 23(4), 104-107.

Comin, D., Ramos, F. M., Zucchi, C., Favretto, J., \& Fachi, C. C. P. (2016). A transparência ativa nos municípios de Santa Catarina: Avaliação do índice de atendimento à Lei de Acesso à Informação e suas determinantes. Revista Catarinense da Ciência Contábil (CRCSC), 15(46), 24-34. doi:10.16930/22377662/rccc.v15n46p24-34

Cruz, C. F. (2010). Transparência da gestão pública municipal: Referenciais teóricos e a situação dos grandes munic ípios brasileiros (Dissertação de mestrado, Universidade Federal do Rio de Janeiro, Rio de Janeiro, RJ).

Cruz, C. F., \& Afonso, L. E. (2018). Gestão fiscal e pilares da Lei de Responsabilidade Fiscal: Evidências em grandes municípios. RAP-Revista de Administração Pública, 52(1), 126-148. doi:10.1590/00347612165847

Cruz, C. F., Ferreira, A. C. S., Silva, L. M., \& Macedo, M. A. S. (2012). Transparência da gestão pública municipal: Um estudo a partir dos portais eletrônicos dos maiores municípios brasileiros. RAP-Revista de Administração Pública, 46(1), 153-176. doi:10.1590/ S0034-76122012000100008

Cruz, C. F., Macedo, M. A. S., \& Sauerbronn, F. F. (2013). Responsabilidade fiscal de grandes municípios brasileiros: Uma análise de suas características. RAP-Revista de Administração Pública, 47(6), 1375-1379. doi:10.1590/S0034-76122013000600003 
Cruz, C. F., Silva, L. M., \& Santos, R. (2009). Transparência da gestão fiscal: Um estudo a partir dos portais eletrônicos dos maiores municípios do Estado do Rio de Janeiro. Contabilidade, Gestão e Governança, 12(3), 102-115.

Federação das Indústrias do Estado do Rio de Janeiro. (2017a). Índice Firjan de Desenvolvimento Municipal. Recuperado de http:// www.firjan.com.br/ifdm/

Federação das Indústrias do Estado do Rio de Janeiro. (2017b). Índice Firjan de Gestão Fiscal. Recuperado de http://www.firjan. com.br/ifgf/

Gujarati, D. N., \& Porter, D. C. (2011). Econometria básica (5a ed.). Porto Alegre, RS: McGraw Hill.

Kelles, M. F. (2010, Junho). Dez anos da Lei de Responsabilidade Fiscal: Da idealidade à efetividade. Revista do Tribunal de Contas do Estado de Minas Gerais, 75(2), 77-96.

Klein, R. H., \& Klein, D. C. B. (2017). Utilização da accountability theory em pesquisas sobre transparência governamental: Abordagens e perspectivas. XLI Encontro da Associação Nacional de Pós-Graduação e Pesquisa em Administração (EnANPAD), São Paulo, SP.

Klering, L. R., Kruel, A. J., \& Stranz, E. (2012). Os pequenos municípios do Brasil: Uma análise a partir de índices de gestão. Análise Revista de Administração da PUCRS, 23(1), 31-44.

Kopits, G., \& Craig, J. (1998). Transparency in government operations [Ocasional Paper 158]. International Monetary Fund. Recuperado de http://www.imf.org/external/pubs/cat/ longres.aspx?sk=2476

Lei Complementar n. 101, de 4 de maio de 2000. (2000). Estabelece normas de finanças públicas voltadas para a responsabilidade na gestão fiscal e dá outras providências. Brasília, DF.

Leite, G., Filho, Santiago, W. P., Otoni, B. S., Veloso, J. T. R., \& Ferreira, K. R. (2015). Efeitos da Lei de Responsabilidade Fiscal nos indicadores de desenvolvimento e de responsabilidade social dos municípios do Estado de Minas Gerais. Revista Mineira de Contabilidade, 16(2), 14-25.

Lock, F. N. (2003). Transparência da gestão municipal através das informações contábeis divulgadas na internet (Dissertação de mestrado, Universidade Federal de Pernambuco, Recife, PE).

Ministério Público Federal. (2017). Ranking nacional da transparência. Recuperado de http://www.rankingdatransparencia.mpf. mp.br/

Oliveira, A. C., \& Santos, N. A. (2017). Transparência passiva nos pequenos municípios brasileiros. XI Congresso Anpcont, Belo Horizonte, MG.

Piccoli, M. R., \& Arpini, M. (2018). A accountability e os motivos que ensejaram a rejeição das contas dos municípios catarinenses. Revista de Administração e Contabilidade, 10(1), 36-47.

Pinho, J. A. G., \& Sacramento, A. R. (2009). 


\section{RELAÇÃO ENTRE A QUALIDADE DA GESTÃo FISCAL E A TRANSPARÊNCIA DOS MUNICÍPIOS BRASILEIROS}

Accountability: Já podemos traduzi-la para o português? RAP-Revista de Administração Pública, 43(6), 1343-1368. doi:10.1590/ S0034-76122009000600006

Platt, O. A., Neto, Cruz, F., Ensslin, S. R., \& Ensslin, L. (2007). Publicidade e transparência das contas públicas: Obrigatoriedade e abrangência desses princípios na administração pública brasileira. Contabilidade Vista \& Revista, 18(1), 75-94.

Reis, A. O., Ferreira, M. A. M., \& Ferreira, M. A. S. (2015). Análise dos fatores determinantes da transparência orçamentária pública em nível nacional. XV Congresso USP Controladoria e Contabilidade, São Paulo, SP.

Ribeiro, C. P. P., \& Zuccolotto, R. (2009). Índice de transparência fiscal das contas públicas dos municípios obtidos em meios eletrônicos de acesso público. XXXIII Encontro da Associação Nacional de Pós-Graduação e Pesquisa em Administração (EnANPAD), São Paulo, SP.

Ribeiro, C. P. P., \& Zuccolotto, R. (2012). Fatores determinantes da transparência na gestão pública dos municípios brasileiros. $\checkmark$ Encontro de Administração Pública e Governo (EnAPG), Salvador, BA.

Rodrigues, M., Júnior, \& Salgueiro, V. (2015). Transparência na gestão fiscal nos municípios do estado do Ceará. Revista Controle - Doutrina e Artigos, 13(1), 47-63.

Sacramento, A. R. S., \& Pinho, J.A. G. (2007). Transparência na administração pública: $O$ que mudou depois da Lei de Responsabilidade Fiscal? Um estudo exploratório em seis municípios da Região Metropolitana de Salvador. Revista de Contabilidade da UFBA, 1(1), 48-61.

Schedler, A. (1999). Conceptualizing accountability. In A. Schedler, L. Diamond, \& M. F. Plattner (Eds.), Self-restraining state: Power and accountability in new democracies (pp. 13-28). London, UK: Lynne Rienner Publishers.

Silva, T. G. (2009). Transparência das contas públicas e Lei de Responsabilidade Fiscal: Detalhamento da despesa em portais de transparência do governo federal (Dissertação de mestrado, Universidade Federal do Rio de Janeiro, Rio de Janeiro, RJ).

Washington, S. P., Karlaftis, M. G., \& Mannering, F. L. (2011). Statistical and econometric methods for transportation data analysis (2nd ed.). Boca Raton, FL: CRC Press.

Wooldridge, J. M. (2006). Introdução à econometria: Uma abordagem moderna. São Paulo, SP: Cengage Learning.

Wright, G. A. (2013). Análise dos fatores determinantes da transparência fiscal ativa nos municípios brasileiros. (Dissertação de mestrado, Universidade de Brasília/UFPB/ UFRN, João Pessoa, PB).

Wright, G. A., \& Paulo, E. (2014). Análise dos fatores determinantes de transparência fiscal ativa nos municípios brasileiros. VIII Congresso Anpcont, Rio de Janeiro, RJ.

Zuccolotto, R., \& Teixeira, M. A. C. (2014). As causas da transparência fiscal: Evidências nos estados brasileiros. Revista Contabilidade \& Finanças, 25(66), 242-254. doi:10.1590/1808-057x201410820 